\title{
T1 Relaxivities of Gadolinium-Based Magnetic Resonance Contrast Agents in Human Whole Blood at 1.5, 3, and $7 \mathrm{~T}$
}

Shen, Yaqi ; Goerner, Frank L ; Snyder, Christopher ; Morelli, John N ; Hao, Dapeng ; Hu, Daoyu ; Li, Xiaoming ; Runge, Val M

\begin{abstract}
OBJECTIVES Calculation of accurate T1 relaxivity (r1) values for gadolinium-based magnetic resonance contrast agents (GBCAs) is a complex process. As such, often referenced r1 values for the GBCAs at $1.5 \mathrm{~T}, 3 \mathrm{~T}$, and $7 \mathrm{~T}$ are based on measurements obtained in media that are not clinically relevant, derived from only a small number of concentrations, or available for only a limited number of GBCAs. This study derives the $\mathrm{r} 1$ values of the 8 commercially available GBCAs in human whole blood at $1.5 \mathrm{~T}, 3 \mathrm{~T}$, and $7 \mathrm{~T}$. MATERIALS AND METHODS Eight GBCAs were serially diluted in human whole blood, at 7 concentrations from 0.0625 to $4 \mathrm{mM}$. A custom-built phantom held the dilutions in air-tight cylindrical tubes maintained at $37 \pm 0.5^{\circ} \mathrm{C}$ by a heat-circulating system. Images were acquired using inversion recovery sequences with inversion times from 30 milliseconds to 10 seconds at $1.5 \mathrm{~T}$ and 3 $\mathrm{T}$ as well as 60 milliseconds to 5 seconds at $7 \mathrm{~T}$. A custom MATLAB program was used to automate signal intensity measurements from the images acquired of the phantom. SigmaPlot was used to calculate T1 relaxation times and, finally, r1. RESULTS Measured r1 values in units of s[BULLET OPERATOR]mM at $1.5 \mathrm{~T}(3 \mathrm{~T} / 7 \mathrm{~T})$ were $3.9 \pm 0.2(3.4 \pm 0.4 / 2.8 \pm 0.4)$ for Gd-DOTA, $4.6 \pm 0.2(4.5 \pm 0.3 / 4.2 \pm$ $0.3)$ for Gd-DO3A-butrol, $4.3 \pm 0.4(3.8 \pm 0.2 / 3.1 \pm 0.4)$ for Gd-DTPA, $6.2 \pm 0.5(5.4 \pm 0.3 / 4.7 \pm$ $0.1)$ for Gd-BOPTA, $4.5 \pm 0.1(3.9 \pm 0.2 / 3.7 \pm 0.2)$ for Gd-DTPA-BMA, $4.4 \pm 0.2(4.2 \pm 0.2 / 4.3 \pm$ $0.2)$ for Gd-DTPA-BMEA, $7.2 \pm 0.2(5.5 \pm 0.3 / 4.9 \pm 0.1)$ for Gd-EOB-DTPA, and $4.4 \pm 0.6(3.5 \pm$ $0.6 / 3.4 \pm 0.1)$ for Gd-HP-DO3A. The agents can be stratified by relaxivity, with a significant additional dependency on field strength. CONCLUSIONS This report quantifies, for the first time, T1 relaxivity for all 8 gadolinium chelates in common clinical use worldwide, at current relevant field strengths, in human whole blood at physiological temperature $\left(37^{\circ} \mathrm{C}\right)$. The measured $\mathrm{r} 1$ values differ to a small degree from previously published values, where such comparisons exist, with the current r1 measurements being that most relevant to clinical practice. The macrocyclic agents, with the exception of Gd-DO3A-butrol, have slightly lower $\mathrm{r} 1$ values when compared with the 2 much less stable linear agents, Gd-DTPA-BMA and Gd-DTPA-BMEA. The 2 agents with hepatobiliary excretion, Gd-EOB-DTPA and Gd-BOPTA, have, at 1.5 and $3 \mathrm{~T}$, substantially higher $\mathrm{r} 1$ values than all other agents.
\end{abstract}

DOI: https://doi.org/10.1097/RLI.0000000000000132

Posted at the Zurich Open Repository and Archive, University of Zurich

ZORA URL: https://doi.org/10.5167/uzh-109077

Journal Article

Published Version

Originally published at: 
Shen, Yaqi; Goerner, Frank L; Snyder, Christopher; Morelli, John N; Hao, Dapeng; Hu, Daoyu; Li, Xiaoming; Runge, Val M (2015). T1 Relaxivities of Gadolinium-Based Magnetic Resonance Contrast Agents in Human Whole Blood at 1.5, 3, and 7 T. Investigative Radiology, 50(5):330-338.

DOI: https://doi.org/10.1097/RLI.0000000000000132 


\title{
T1 Relaxivities of Gadolinium-Based Magnetic Resonance Contrast Agents in Human Whole Blood at 1.5, 3, and $7 \mathrm{~T}$
}

\author{
Yaqi Shen, MD, PhD, * Frank L. Goerner, PhD, $† \$$ Christopher Snyder, BS, † John N. Morelli, MD, /I \\ Dapeng Hao, MD, ๆ Daoyu Hu, MD, * Xiaoming Li, MD, * and Val M. Runge, MD\#
}

\begin{abstract}
Objectives: Calculation of accurate T1 relaxivity $\left(r_{1}\right)$ values for gadoliniumbased magnetic resonance contrast agents (GBCAs) is a complex process. As such, often referenced $r_{1}$ values for the GBCAs at $1.5 \mathrm{~T}, 3 \mathrm{~T}$, and $7 \mathrm{~T}$ are based on measurements obtained in media that are not clinically relevant, derived from only a small number of concentrations, or available for only a limited number of GBCAs. This study derives the $r_{1}$ values of the 8 commercially available GBCAs in human whole blood at $1.5 \mathrm{~T}, 3 \mathrm{~T}$, and $7 \mathrm{~T}$.

Materials and Methods: Eight GBCAs were serially diluted in human whole blood, at 7 concentrations from 0.0625 to $4 \mathrm{mM}$. A custom-built phantom held the dilutions in air-tight cylindrical tubes maintained at $37 \pm 0.5^{\circ} \mathrm{C}$ by a heatcirculating system. Images were acquired using inversion recovery sequences with inversion times from 30 milliseconds to 10 seconds at $1.5 \mathrm{~T}$ and $3 \mathrm{~T}$ as well as 60 milliseconds to 5 seconds at $7 \mathrm{~T}$. A custom MATLAB program was used to automate signal intensity measurements from the images acquired of the phantom. SigmaPlot was used to calculate T1 relaxation times and, finally, $r_{1}$.

Results: Measured $r_{1}$ values in units of $\mathrm{s}^{-1} \cdot \mathrm{mM}^{-1}$ at $1.5 \mathrm{~T}(3 \mathrm{~T} / 7 \mathrm{~T})$ were $3.9 \pm 0.2(3.4 \pm 0.4 / 2.8 \pm 0.4)$ for Gd-DOTA, $4.6 \pm 0.2(4.5 \pm 0.3 / 4.2 \pm 0.3)$ for Gd-DO3A-butrol, $4.3 \pm 0.4(3.8 \pm 0.2 / 3.1 \pm 0.4)$ for Gd-DTPA, $6.2 \pm 0.5$ $(5.4 \pm 0.3 / 4.7 \pm 0.1)$ for Gd-BOPTA, $4.5 \pm 0.1(3.9 \pm 0.2 / 3.7 \pm 0.2)$ for $\mathrm{Gd}-$ DTPA-BMA, $4.4 \pm 0.2(4.2 \pm 0.2 / 4.3 \pm 0.2)$ for Gd-DTPA-BMEA, $7.2 \pm 0.2$ $(5.5 \pm 0.3 / 4.9 \pm 0.1)$ for Gd-EOB-DTPA, and $4.4 \pm 0.6(3.5 \pm 0.6 / 3.4 \pm 0.1)$ for Gd-HP-DO3A. The agents can be stratified by relaxivity, with a significant additional dependency on field strength.

Conclusions: This report quantifies, for the first time, T1 relaxivity for all $8 \mathrm{gad}-$ olinium chelates in common clinical use worldwide, at current relevant field strengths, in human whole blood at physiological temperature $\left(37^{\circ} \mathrm{C}\right)$. The measured $r_{1}$ values differ to a small degree from previously published values, where such comparisons exist, with the current $r_{1}$ measurements being that most relevant to clinical practice. The macrocyclic agents, with the exception of GdDO3A-butrol, have slightly lower $r_{1}$ values when compared with the 2 much less stable linear agents, Gd-DTPA-BMA and Gd-DTPA-BMEA. The 2 agents with hepatobiliary excretion, Gd-EOB-DTPA and Gd-BOPTA, have, at 1.5 and $3 \mathrm{~T}$, substantially higher $r_{1}$ values than all other agents.
\end{abstract}

Key Words: relaxivity, MRI contrast media, gadolinium, whole blood, field strength dependence, $1.5 \mathrm{~T}, 3 \mathrm{~T}, 7 \mathrm{~T}$, comparative studies

(Invest Radiol 2015;00: 00-00)

Received for publication September 8, 2014; and accepted for publication, after revision, November 18, 2014.

From the *Department of Radiology, Tongji Hospital, Tongji Medical College, Huazhong University of Science and Technology, Wuhan, Hubei, People's Republic of China; †Department of Radiology, University of Texas Medical Branch, Galveston; †Department of Pediatric Radiology, Texas Children's Hospital; §Department of Radiology, Baylor College of Medicine, Houston, TX; \|The Russell H. Morgan Department of Radiology and Radiological Science, Johns Hopkins University, Baltimore, MD; -Department of Radiology, The Affiliated Hospital of Medical College of Qingdao University, Qingdao, People's Republic of China; and \#Institute for Diagnostic and Interventional Radiology, Clinics for Neuroradiology and Nuclear Medicine, University Hospital Zurich, Zurich, Switzerland.

Conflicts of interest and sources of funding: Supported in part by Bracco Imaging (Milan, Italy) and the National Scientific Foundation of China (no. 81320108013 and no. 31170899).

The authors report no conflicts of interest.

Reprints: Xiaoming Li, MD, Department of Radiology, Tongji Hospital, Tongji Medical College, Huazhong University of Science and Technology, Wuhan, Hubei, People's Republic of China. E-mail: lilyboston2002@163.com.

Copyright (C) 2015 Wolters Kluwer Health, Inc. All rights reserved. ISSN: 0020-9996/15/0000-0000
G adopentetate dimeglumine (Magnevist, Gd-DTPA) was the first gadolinium (Gd)-based magnetic resonance (MR) contrast agent (GBCA) approved for clinical use, which occurred in 1988. To date, 9 GBCAs have been approved for use in Europe and the United States. ${ }^{1,2}$ The additional 8 include Gd-BOPTA (gadobenate dimeglumine, MultiHance), Gd-DO3A-Butrol (gadobutrol, Gadovist/ Gadavist), Gd-DOTA (gadoterate meglumine, Dotarem), Gd-DTPABMA (gadodiamide, Omniscan), Gd-DTPA-BMEA (gadoversetamide, OptiMARK), Gd-EOB-DTPA (gadoxetic acid disodium, Primovist/ Eovist), Gd-HP-DO3A (gadoteridol, ProHance), and MS-325 (gadofosveset trisodium, Ablavar). In clinical practice, the GBCAs are most commonly used to improve detection and differentiation of pathological lesions and to visualize the vasculature in MR angiography applications.

Relaxivity is a critical parameter in determining the relative efficacy of the GBCAs. Relaxivity is defined as the change in reciprocal relaxation time per unit concentration of Gd chelate. T1 relaxivity, known as $r_{1}$, is influenced by many variables including magnetic field strength, temperature, environmental conditions, and protein concentration. ${ }^{1,3-5}$

Previous in vitro studies have examined the relative relaxivities of the Gd chelates. However, these have been incomplete (not including all relevant field strengths or deriving calculations from a wide range of concentrations), inapplicable to human imaging (not performed in human whole blood at physiologic temperatures), or failing to account for nonlinear $1 / \mathrm{T} 1 \mathrm{vs}[\mathrm{Gd}]$ when proteins are present for the proteinbinding agents. ${ }^{3,4,6-10}$ This study aimed to address these limitations by determining the $\mathrm{T} 1$ relaxivity values across a wide range of concentrations at $1.5 \mathrm{~T}, 3 \mathrm{~T}$, and $7 \mathrm{~T}$ for the 8 primary, commercially available GBCAs (excluding MS-325, which is strongly protein bound, seldom used in clinical work in most of the world, and no longer commercially available in Europe) in human whole blood at $37^{\circ} \mathrm{C}$.

\section{MATERIALS AND METHODS}

\section{Preparation of Human Whole Blood Samples}

Whole human blood was acquired from a commercial supplier (Valley Biomedical Products \& Services, Inc). Owing to $7 \mathrm{~T}$ scanners not being widely available, the experiments were performed at 2 different sites: one housing the 1.5 and $3 \mathrm{~T}$ scanners (site 1) and the other housing the $7 \mathrm{~T}$ scanner (site 2). This also required a different human blood sample to be used at each respective site because it was not possible to preserve the first sample set for use at the second site. For standardization purposes, hematocrit fractions (Hct) of each sample set were measured before dilution (site 1: Hct was $50 \pm 0.5$; site 2: Hct was $45 \pm 0.5$ ). To ensure that relaxivity results were not affected by the different Hct concentrations within the 2 different sample sets, measurements at $3 \mathrm{~T}$, further described below, were performed on each sample set. After the Hct measurement, 8 commercially available GBCAs (Table 1) were serially diluted in human whole blood (Valley Biomedical Products \& Services, Inc). The metal concentration of $\mathrm{Gd}$ in the same bottles that were used for dilution was verified using the inductively coupled plasma optical emission spectrometer (ICP-OES) technique (PerkinElmer, mod. OPTIMA 2100 DV) after all experiments were completed (Table 2). The ICP-OES was performed with a wavelength of observation of $342.247 \mathrm{~nm}$, and the measurements were based on a calibration curve 
TABLE 1. Investigated Gd-Based MR Contrast Agents

\begin{tabular}{lccc}
\hline Chemical Name & Generic Name (INN) & Trade Name & Manufacturer \\
\hline Gd-BOPTA & Gadobenate dimeglumine & MultiHance & Bracco \\
Gd-DO3A-butrol & Gadobutrol & Gadovist/Gadavist & Bayer HealthCare \\
Gd-DOTA & Gadoterate meglumine & Dotarem & Guerbet \\
Gd-DTPA & Gadopentetate dimeglumine & Magnevist & Bayer HealthCare \\
Gd-DTPA-BMA & Gadodiamide & Omniscan & GE-Healthcare \\
Gd-DTPA-BMEA & Gadoversetamide & OptiMARK & Covidien \\
Gd-EOB-DTPA & Gadoxetic acid disodium & Primovist/Eovist & Bayer HealthCare \\
Gd-HP-DO3A & Gadoteridol & ProHance & Bracco \\
\hline
\end{tabular}

Gd indicates gadolinium; INN, International Nonproprietary Names; MR, magnetic resonance.

acquired between 0.2 and $5.0 \mu \mathrm{g} / \mathrm{mL}$. Each GBCA sample was measured 3 times independently at different dilution factors. The ICP-OES system settings were as follows: axial view, internal standard Rh 343.489 nm; 1300W, Neb 0.7, Aux 0.2, Myramist nebulizer, Quartz cyclonic, $\mathrm{Al} 2 \mathrm{O} 3$ injector.

An initial preliminary experiment demonstrated that the presence of citrate did not affect GBCA relaxation times; thus, the specimens were diluted with sodium citrate added as an anticoagulant. Samples were stored at $4 \pm 0.05^{\circ} \mathrm{C}$ until the day of scan. The half-life of human serum is approximately 20 days; thus, all measurements were performed less than 10 days within preparation of the whole blood samples.

All solutions were placed into glass NMR tubes $(5.5 \times 0.5 \mathrm{~cm}$; Wilmad-Lab Glass Company). Both ends were sealed with silicone to prevent evaporation, leakage, and contamination. Each tube was used only once and was disposed of at the end of the measurement. The samples were serially diluted with GBCA to achieve final concentrations of $0,0.0625,0.125,0.25,0.5,1,2$, and $4 \mathrm{mM} \mathrm{GBCA}$ at $\mathrm{pH}=7.4 \pm 0.05$. The samples were then loaded into the subsequently described phantom and scanned at $1.5 \mathrm{~T}, 3 \mathrm{~T}$, and $7 \mathrm{~T}$. To minimize the error due to sedimentation of red blood cells, all the tubes were gently agitated before and during the MR image acquisitions.

Properties of the samples used for 1.5 and $3 \mathrm{~T}$ measurements at site 1 were as follows: total protein concentration of $6.5 \pm 0.05 \mathrm{~g} \cdot \mathrm{dL}^{-1}$, albumin of $4.3 \pm 0.05 \mathrm{~g} \cdot \mathrm{dL}^{-1}$, and Hct of $50 \pm 0.5$. Properties of the samples used for $7 \mathrm{~T}$ measurements at site 2 were as follows: total protein concentration of $6.3 \pm 0.05 \mathrm{~g} \cdot \mathrm{dL}^{-1}$, albumin of $4.2 \pm 0.05 \mathrm{~g} \cdot \mathrm{dL}^{-1}$, and Hct of $45 \pm 0.5$.

For quality control purposes, subsets of each of the 2 sample sets were imaged on a $3 \mathrm{~T}$ scanner and the results were compared. Concentrations used for these subset quality control measurements were from 0.25 to $4 \mathrm{mM}$.

\section{Custom-Built Relaxivity Phantom}

All the tubes were placed parallel in a custom-designed acrylic holder in which 3 plastic screws were used to sandwich the tubes, holding them in place between 2 plastic plates (Fig. 1). Each holder contained 2 sets of GBCAs $(0.0625-4 \mathrm{mM})$ and a tube without any GBCA as a control. The holder was put in the center of an air-tight cylindrical acrylic container with a removable cap. The container was equipped with 2 equal-sized flanges to allow for water to flow in and out as well as an additional small hole in which a fiber optic probe for temperature measurement was inserted (site 1: Essential, In vivo, FL; site 2: Fluoroptic temperature probe, LumaSense Technologies, CA). To control temperature, plastic tubing was attached to the flanges. The tubing was then connected to a hot water bath (Cole-Parmer Company, IL). This allowed for warm water to flow in and out of the phantom. The water exchange inside the phantom was manually controlled to maintain a narrow temperature range for the samples $\left(37 \pm 0.5^{\circ} \mathrm{C}\right)$.
During scan acquisition, the water only circulated outside the phantom to avoid artifacts from the motion of water.

\section{Imaging Protocol}

Measurements were performed at $1.5 \mathrm{~T}$ (Avanto; Siemens Healthcare, Erlangen, Germany) on a clinical whole-body MR scanner with an 8-channel knee coil for signal acquisition, a $3 \mathrm{~T}$ (Skyra; Siemens Healthcare, Erlangen, Germany) MR system with a Tx/Rx 15-channel knee coil, and a $7 \mathrm{~T}$ (Philips Healthcare, Cleveland, $\mathrm{OH}$ ) preclinical MR scanner with a Tx/Rx 32-channel head coil. Additional measurements were performed at $3 \mathrm{~T}$ (Verio; Siemens Healthcare, Erlangen, Germany) with a Tx/Rx 15-channel knee coil. For each measurement, a single 5 -mm-thick slice was obtained perpendicular to the long axis of the tubes. The tubes were positioned parallel to $\mathrm{B}_{0}$ along the $z$ axis in the magnet.

The longitudinal relaxation rate constant $R_{1}$ was calculated as the inverse of T1 relaxation time. Successive inversion recovery turbo spinecho sequences with varying inversion times (TIs) were used to measure the longitudinal relaxation rate constant $R_{1}$.

\section{Protocol for 1.5 and $3 \mathrm{~T}$}

Two different protocols (with different ranges of TI and repetition time [TR]) aimed at evaluation of different ranges of T1 times were used in this study. This was done to account for the large range of T1 times of the dilutions and for the necessity of having a TR at least 3 times the expected $\mathrm{T} 1 .{ }^{11}$ When looking at the results in retrospect, it was deemed unnecessary to perform both protocols because our results show no statistically significance difference. Nonetheless, we chose to report results from both protocols here. The $1.5 \mathrm{~T}$ and $3 \mathrm{~T}$ samples were

TABLE 2. ICP-OES Results of Investigated Gd-Based MR Contrast Agents

\begin{tabular}{lccc}
\hline Chemical name & $\begin{array}{c}\text { Formulation } \\
(\mathbf{M})\end{array}$ & $\begin{array}{c}\text { ICP-OES } \\
\text { (M) }\end{array}$ & $\begin{array}{c}\text { Percentage Variation } \\
\text { From Reference Value }\end{array}$ \\
\hline Gd-BOPTA & 0.5 & $0.47 \pm 0.01$ & -5.81 \\
Gd-DO3A-butrol & 1 & $0.95 \pm 0.03$ & -4.91 \\
Gd-DOTA & 0.5 & $0.48 \pm 0.01$ & -4.64 \\
Gd-DTPA & 0.5 & $0.48 \pm 0.01$ & -3.61 \\
Gd-DTPA-BMA & 0.5 & $0.49 \pm 0.01$ & -2.33 \\
Gd-DTPA-BMEA & 0.5 & $0.47 \pm 0.00$ & -5.23 \\
Gd-EOB-DTPA & 0.25 & $0.23 \pm 0.00$ & -6.56 \\
Gd-HP-DO3A & 0.5 & $0.48 \pm 0.01$ & -3.84
\end{tabular}

Gd indicates gadolinium; ICP-OES, inductively coupled plasma optical emission spectrometer technique; MR, magnetic resonance. 

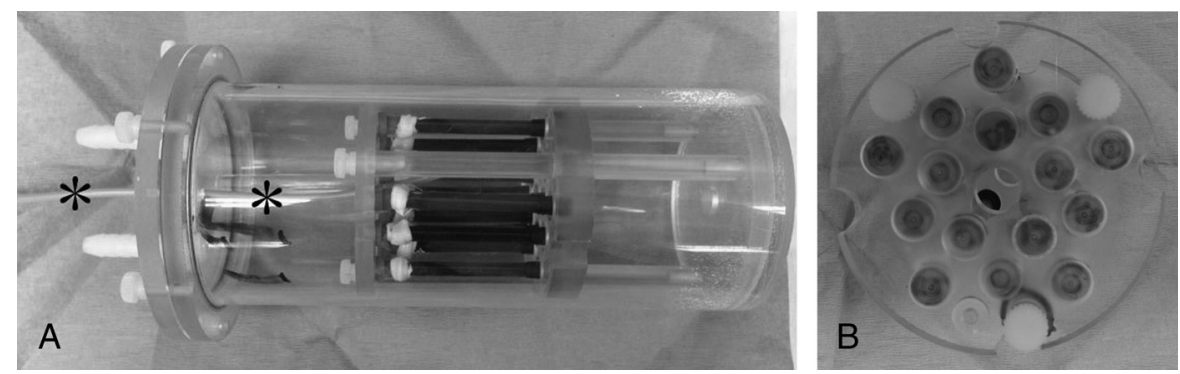

FIGURE 1. Custom-built holder and phantom. All the tubes are placed parallel to each other in a custom-design acrylic holder, using plastic screws to sandwich the tubes and hold them in place. The holder was placed in the center of phantom with a removable cap. There was 1 hole in the center in which to place a temperature probe (*). The holder is shown in longitudinal (A) and transverse (B) views.

imaged with both of the following protocols, aimed at measuring the T1 values in parentheses:

\section{Protocol 1 (for the Assessment of T1 Values From Approximately 50 to 600 Milliseconds)}

The TIs are the following: 30, 60, 90, 120, 150, 250, 400, 600, $800,1200,1600,2000,2400,2800$, and 3200 milliseconds.

The TR is 1500 milliseconds + TI.

\section{Protocol 2 (for the Assessment of T1 Values Longer Than 600 milliseconds)}

The TIs are the following: $60,120,250,400,800,1600,2400$, 3200, 5000, 7000, and 10,000 milliseconds.

The TR is 4000 milliseconds + TI.

For both protocols, the following parameters were held constant: echo time, 15 milliseconds ( $3 \mathrm{~T}) / 11$ milliseconds (1.5 T); matrix, $256 \times$ 256; field of view, $80 \times 80 \mathrm{~mm}^{2}$; slice thickness, $5 \mathrm{~mm}$; and in-plane spatial resolution, 3.2 pixels per millimeter.

\section{Protocol for $7 \mathrm{~T}$}

For $7 \mathrm{~T}$, the imaging protocol was optimized for the higher field strength. After a preliminary experiment on 2 of the 8 GBCAs, specific TRs and TIs were chosen to cover the whole concentration range within a reasonable scan time that allowed for temperature to be maintained as well as to maintain image quality equivalent to that at $1.5 \mathrm{~T}$ and $3 \mathrm{~T}$.

\section{Protocol}

The TIs are the following: $60,80,120,160,220,350,500,650$, $900,1800,3000$, and 5000 milliseconds.

The TR is 3000 milliseconds $+\mathrm{TI}$.
The echo time is 10 milliseconds; matrix, $320 \times 320$; field of view, $90 \times 90 \mathrm{~mm}^{2}$; slice thickness, $5 \mathrm{~mm}$; and in-plane spatial resolution, 3.6 pixels per millimeter.

\section{Calculation of Longitudinal Relaxation Rate Constant $R_{\mathbf{1}}$}

Once the images were acquired, regions of interest (ROIs) were automatically placed using custom-designed MATLAB code to detect the mean signal intensities (SIs) of the sample within the tubes. The code was designed to only include pixels within the sample and to eliminate partial volume artifact, resulting in ROIs of 100 pixels for $3 \mathrm{~T} / 1.5 \mathrm{~T}$ and 140 pixels for $7 \mathrm{~T}$. The MATLAB code produced mean ROI measurements and an image that showed the ROI placements. All images were manually observed for proper MATLAB ROI placement. The ROIs that were observed to include susceptibility artifact were then manually placed, and this measurement was used in subsequent calculations. Since the signal intensities were all positive, because the scans provided magnitude data only, the inflection point was chosen according to the best fit curve equation (1). ${ }^{12}$ Thus, positive numbers below the inflection point along the $x$ axis were converted to negative. $R_{1}$ values were obtained using equation (1) and a 3-parameter curve fitting tool (Sigma Plot 12.0). After plotting SIs versus TIs, an exponential curve was constructed to characterize $R_{1}$ and the fitting error was used to describe the uncertainty of $R_{1}$ (Fig. 2).

$$
S I_{T I}=A+B e^{-T I R_{1}}
$$

The $7 \mathrm{~T}$ Philips system had a postprocessing algorithm that scaled the pixel values of each collected MR image. This made the images unacceptable for relaxation measurements, and the raw SI values had to be restored. Restoration was accomplished using equation (2),

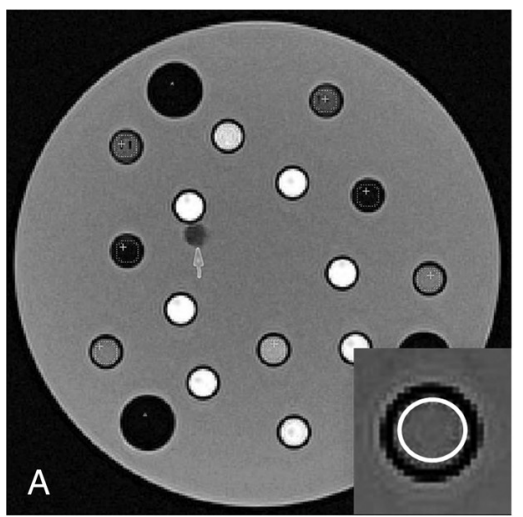

Signal Intensity vs. Inversion Time-Protocol 1 at $3 \mathrm{~T}$

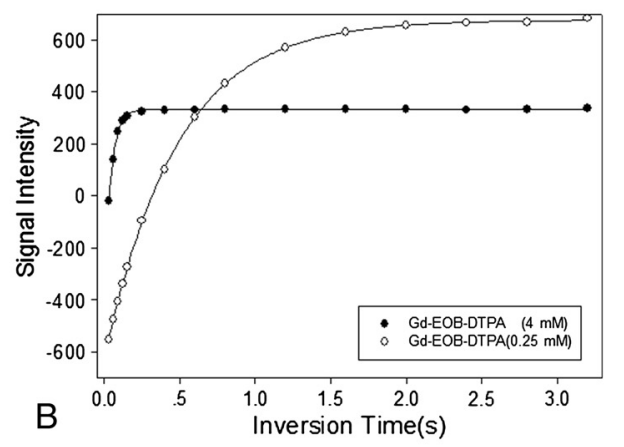

Gd-EOB-DTPA at 3 T Concentration $(\mathrm{mM})$ vs. $\mathrm{R}_{1}\left(\mathrm{~s}^{-1}\right)$

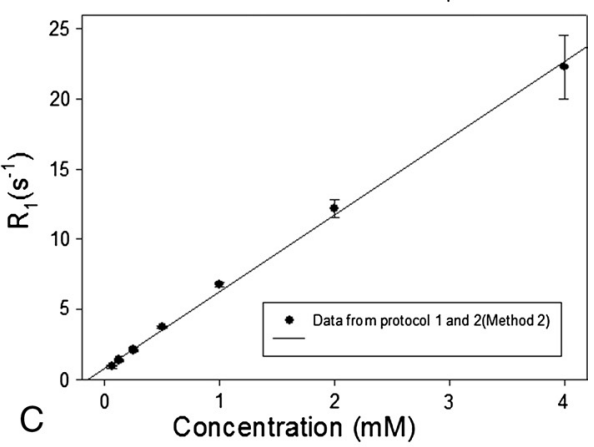

FIGURE 2. Analysis of data. A, Regions of interests were automatically placed by the MATLAB program, signified by white rings (at $3 \mathrm{~T}$, $\mathrm{TI}=60$, zoomed in the right corner). Pixels inside the rings were measured. The temperature probe is denoted by the arrow. B, Mean SI values were used to plot T1 relaxation curves. This particular example shows curves for Gd-EOB-DTPA at a concentration of $0.25 \mathrm{mM}$ and $4 \mathrm{mM}$ at $3 \mathrm{~T}$. C, Plotting $R_{1}$ against concentration to calculate the $r_{1}$ (at 3 T for Gd-EOB-DTPA). 

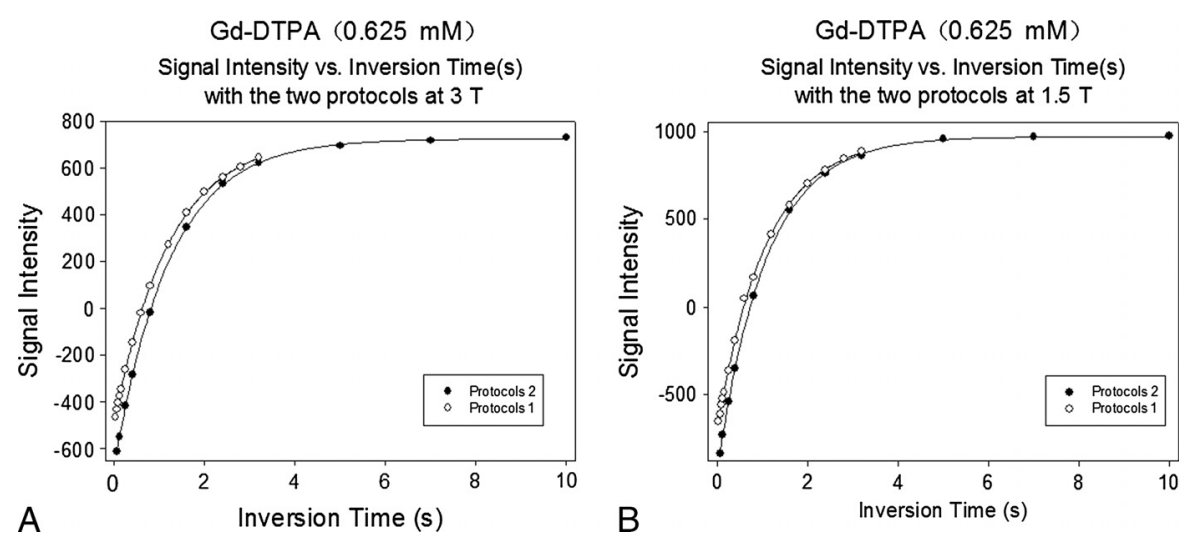

FIGURE 3. Graph illustrating Sls versus Tls for the 2 protocols used to measure $r_{1}$ relaxivity at $1.5 \mathrm{~T}$ and $3 \mathrm{~T}$. This example is of Gd-DOTA evaluated at a concentration of $0.0625 \mathrm{mM}$ at $3 \mathrm{~T}$, with protocols 1 and $2(\mathrm{~A})$ as well as at $1.5 \mathrm{~T}$ with protocols 1 and 2 (B).

scale slope and intercept are Philips tags included in the Digital Imaging and Communications in Medicine file for each image, and all the intercepts were 0 in this study.

$$
S I_{7 T}=\frac{S I_{\text {Matlab }}-\text { Intercept }}{\text { Scale slope }}
$$

\section{Calculation of Relaxivities}

Relaxivity is the second-order rate constant defining the ability of any GBCA to catalyze water proton relaxation. ${ }^{1}$

The $r_{1}$ values were calculated from equation (3), where $R_{1(\mathrm{c})}$ was the relaxation rate constant of the contrast agent at concentration $\mathrm{C}$, and $R_{1(0)}$ denoted the relaxation rate constant of whole blood. Respective error bars were attached to the plot of $R_{1}$ versus concentration. The curve fit error was used to estimate the errors of $r_{1}$ (Fig. 3 ).

$$
r_{1}=\frac{R_{1(c)}-R_{1(0)}}{C}
$$

For $1.5 \mathrm{~T}$ and $3 \mathrm{~T}$ evaluations, measurements from 2 protocols were used in 3 different ways to calculate $r_{1}$. This was done to ensure that we had the most accurate relaxivity values possible and to ensure that the 2 different protocols did not significantly impact our results.

- Method 1: $r_{1}$ was calculated by data gained from protocol 1 only.

- Method 2: $r_{1}$ was calculated by combined data from protocol 1 and protocol 2 (concentration range from $0.0625-0.25 \mathrm{mM}$ ).
- Method 3: $r_{1}$ was calculated by data gained from protocol 1 over the concentration range from 0.5 to $4 \mathrm{mM}$ and from protocol 2 over the concentration range from 0.0625 to $0.25 \mathrm{mM}$.

\section{Statistical Analysis}

For the concentration ranges of 0.25 to $0.0625 \mathrm{mM}$ and bloodonly controls, longitudinal relaxation rate constants $R_{1}$ that were calculated using protocol 1 were compared with those calculated from protocol 2 using a one-way repeat measure analysis of variance (SigmaPlot 12.0). For those that failed in the equal variance test, test execution ended by ranks. The longitudinal relaxation rate constant $R_{1}$ of human whole blood from site 1 , which were calculated from protocol 1 , were compared with site 2 using a rank sum test (SigmaPlot 12.0).

\section{RESULTS}

Results of ICP-OES for the 8 GBCAs are shown in Table 2. All of them were slightly lower than the label.

\section{Comparison Between the Longitudinal Relaxation Rate Constant $R_{1}$ Calculations From the 2 Imaging Protocols at $1.5 \mathrm{~T}$ and $3 \mathrm{~T}$}

At $1.5 \mathrm{~T}$, there was no statistically significant difference seen between $R_{1}$ values calculated using protocol 1 or $2(P>0.05)$ aside from a borderline statistically significant difference found for Gd-DTPA $(P=$ 0.049 ). These results are summarized in Table 3 . No statistically significant differences between $R_{1}$ calculations between the 2 protocols were

\begin{tabular}{|c|c|c|c|c|c|c|c|c|}
\hline \multirow[b]{2}{*}{ Concentration (mM) } & \multicolumn{3}{|c|}{ Protocol 1} & \multicolumn{3}{|c|}{ Protocol 2} & \multirow[t]{2}{*}{$\boldsymbol{F}$} & \multirow[t]{2}{*}{$\boldsymbol{P}$} \\
\hline & 0.25 & 0.125 & 0.0625 & 0.25 & 0.125 & 0.0625 & & \\
\hline Gd-BOPTA & $2.53 \pm 0.09$ & $1.71 \pm 0.07$ & $1.18 \pm 0.04$ & $2.52 \pm 0.06$ & $1.65 \pm 0.07$ & $1.18 \pm 0.03$ & 2.335 & 0.266 \\
\hline Gd-DO3A-butrol & $2.04 \pm 0.07$ & $1.30 \pm 0.05$ & $0.98 \pm 0.05$ & $2.01 \pm 0.08$ & $1.30 \pm 0.05$ & $1.01 \pm 0.02$ & 0.008 & 0.936 \\
\hline Gd-DOTA & $1.77 \pm 0.06$ & $1.16 \pm 0.05$ & $0.93 \pm 0.08$ & $1.79 \pm 0.12$ & $1.17 \pm 0.05$ & $0.93 \pm 0.03$ & 2.133 & 0.282 \\
\hline Gd-DTPA & $2.19 \pm 0.06$ & $1.43 \pm 0.05$ & $1.08 \pm 0.04$ & $2.22 \pm 0.11$ & $1.44 \pm 0.08$ & $1.11 \pm 0.03$ & 19.104 & 0.049 \\
\hline Gd-DTPA-BMA & $1.91 \pm 0.04$ & $1.34 \pm 0.05$ & $1.01 \pm 0.05$ & $1.97 \pm 0.07$ & $1.34 \pm 0.06$ & $1.02 \pm 0.03$ & 2.292 & 0.269 \\
\hline Gd-DTPA-BMEA & $2.06 \pm 0.05$ & $1.34 \pm 0.05$ & $0.99 \pm 0.05$ & $2.02 \pm 0.05$ & $1.34 \pm 0.04$ & $0.99 \pm 0.03$ & 0.650 & 0.505 \\
\hline Gd-EOB-DTPA & $2.48 \pm 0.07$ & $1.69 \pm 0.08$ & $1.15 \pm 0.05$ & $2.42 \pm 0.06$ & $1.65 \pm 0.04$ & $1.14 \pm 0.03$ & 5.957 & 0.135 \\
\hline Gd-HP-DO3A & $2.31 \pm 0.06$ & $1.70 \pm 0.07$ & $1.36 \pm 0.05$ & $2.32 \pm 0.05$ & $1.65 \pm 0.07$ & $1.34 \pm 0.05$ & 1.570 & 0.337 \\
\hline
\end{tabular}

TABLE 3. Comparison of $R_{1}\left(\mathrm{~s}^{-1}\right)$ of GBCAs Using the 2 Protocols at $1.5 \mathrm{~T}$

GBCAs indicates gadolinium-based magnetic resonance contrast agents. 
TABLE 4. Comparison of $R_{1}\left(s^{-1}\right)$ of GBCAs Using the 2 Protocols at $3 \mathrm{~T}$

\begin{tabular}{|c|c|c|c|c|c|c|c|c|}
\hline \multirow[b]{2}{*}{ Concentration (mM) } & \multicolumn{3}{|c|}{ Protocol 1} & \multicolumn{3}{|c|}{ Protocol 2} & \multirow[t]{2}{*}{$\boldsymbol{F}$} & \multirow[t]{2}{*}{$P$} \\
\hline & 0.25 & 0.125 & 0.0625 & 0.25 & 0.125 & 0.0625 & & \\
\hline Gd-BOPTA & $2.06 \pm 0.02$ & $1.36 \pm 0.01$ & $0.97 \pm 0.03$ & $2.33 \pm 0.06$ & $1.32 \pm 0.07$ & $0.96 \pm 0.02$ & 0.531 & 0.542 \\
\hline Gd-DO3A-butrol & $1.83 \pm 0.01$ & $1.16 \pm 0.03$ & $0.84 \pm 0.04$ & $1.72 \pm 0.15$ & $1.14 \pm 0.03$ & $0.84 \pm 0.05$ & & $1^{*}$ \\
\hline Gd-DOTA & $1.61 \pm 0.07$ & $1.05 \pm 0.03$ & $0.82 \pm 0.03$ & $1.51 \pm 0.06$ & $1.03 \pm 0.02$ & $0.82 \pm 0.03$ & 2.589 & 0.249 \\
\hline Gd-DTPA & $1.78 \pm 0.02$ & $1.26 \pm 0.04$ & $0.96 \pm 0.02$ & $1.75 \pm 0.07$ & $1.23 \pm 0.05$ & $0.98 \pm 0.03$ & 0.895 & 0.444 \\
\hline Gd-DTPA-BMA & $1.71 \pm 0.01$ & $1.19 \pm 0.03$ & $0.91 \pm 0.03$ & $1.67 \pm 0.10$ & $1.17 \pm 0.05$ & $0.90 \pm 0.02$ & 9.318 & 0.093 \\
\hline Gd-DTPA-BMEA & $1.86 \pm 0.02$ & $1.20 \pm 0.03$ & $0.86 \pm 0.03$ & $1.87 \pm 0.11$ & $1.19 \pm 0.03$ & $0.87 \pm 0.03$ & 0.0717 & 0.814 \\
\hline Gd-EOB-DTPA & $2.08 \pm 0.05$ & $1.41 \pm 0.03$ & $0.92 \pm 0.11$ & $2.15 \pm 0.14$ & $1.36 \pm 0.01$ & $0.95 \pm 0.03$ & 0.153 & 0.733 \\
\hline Gd-HP-DO3A & $1.75 \pm 0.02$ & $1.51 \pm 0.02$ & $1.18 \pm 0.04$ & $1.64 \pm 0.10$ & $1.51 \pm 0.05$ & $1.19 \pm 0.03$ & 0.791 & 0.468 \\
\hline
\end{tabular}

*The equal variance test failed, and test execution was ended by ranks.

GBCA indicates gadolinium-based magnetic resonance contrast agents.

found for any contrast agent at $3 \mathrm{~T}(P>0.1)$. These results are summarized in Figure 3 and Table 4.

\section{Comparison Between the Longitudinal Relaxation Rate Constants $R_{1}$ of Human Whole Blood at $1.5 \mathrm{~T}, 3 \mathrm{~T}$, and $7 \mathrm{~T}$}

The longitudinal relaxation rate constants $R_{1}$ of human whole blood were calculated at the 3 field strengths with comparisons of the $R_{1}$ values obtained based on the protocol used. At both 1.5 and $3 \mathrm{~T}$, there were statistically significant differences in the calculated $R_{1}$ based on the protocol used (for $1.5 \mathrm{~T}, P=0.002$; for $3 \mathrm{~T}, P=0.027$ ). For each site, 4 control samples were used. This is shown in Table 5 and Figure 4. There was no statistically significant difference seen between $R_{1}$ values calculated with protocol 1 of human whole blood used in the 2 sites at $3 \mathrm{~T}$.

\section{$r_{1}$ Values of the 8 Compared GBCAs}

The $r_{1}$ relaxivities of the 8 compared GBCAs were calculated for the 3 methods at 1.5 and 3 T. These values are shown in Table 6 and Figure 5. An overview of the 8 agents and their respective $r_{1}$ relaxivities at $1.5,3$, and $7 \mathrm{~T}$ is presented in Figure 6.

\section{Comparison of $r_{1}$ Values for the 8 GBCAs Diluted in Human Whole Blood With Previous Results}

Table 7 details the results from the present study for measurement of $r_{1}$ relaxivities for the $8 \mathrm{GBCAs}$ alongside previous results published in the scientific literature. On the basis of our calculated standard deviations and the results from all 3 methods, we present the values in Table 7 as our most accurate measurement of $r_{1}$ for all GBCAs. These results were obtained using method 2 .

\section{DISCUSSION}

This study calculated the $r_{1}$ relaxivities of the 8 available GBCAs at $1.5,3$, and $7 \mathrm{~T}$ from 7 different concentrations in human whole blood at $37^{\circ} \mathrm{C}$. Relaxivity values represent the GBCAs' ability to shorten $\mathrm{T} 1$ times. Gadolinium-based MR contrast agents with high relaxivity values shorten tissue T1 times more efficaciously. They are divided in terms of their ability to bind serum proteins into the nonprotein-binding GBCAs (Gd-DTPA, Gd-DOTA, Gd-HP-DO3A, Gd-BT-DO3A, GdDTPA-BMA, and Gd-DTPA-BMEA), the weakly protein-binding GBCAs (Gd-BOPTA, Gd-EOB-DTPA), and the strongly proteinbinding Gd agent (MS-325). ${ }^{13}$ All GBCAs approved in the United States and Europe other than MS-325 are assessed in the present work. In doing so, limitations of several previous studies are addressed because variations in experimental methods result in variations in calculated $r_{1}$ values. Although several previous works have been performed on this topic, none of them perform calculations in human whole blood. In a study by Rohrer et al, ${ }^{8}$ all available MR contrast agents were compared at $0.47 \mathrm{~T}, 1.5 \mathrm{~T}, 3 \mathrm{~T}$, and $4.7 \mathrm{~T}$ at $37^{\circ} \mathrm{C}$, but only 2 concentrations $(0.25$ and $0.5 \mathrm{mmol} / \mathrm{L})$ were used for the calculation. Further, MR contrast agents were diluted in water, bovine plasma, and canine blood to measure relaxivities, rather than whole human blood. Pintaske et $\mathrm{al}^{3}$ performed a similar comparison in human plasma and over a wider concentration range $(0.01-64 \mathrm{mmol} / \mathrm{L})$ at $0.2 \mathrm{~T}, 1.5 \mathrm{~T}$, and $3 \mathrm{~T}$. However, only 3 GBCAs (Gd-DTPA, Gd-BT-DO3A, and Gd-BOPTA) were evaluated. The study of Noebauer-Huhmann et $\mathrm{al}^{14}$ was the first performed at $7 \mathrm{~T}$. In this work, T1 relaxivity values of 8 GBCAs commercially available in Europe at $3 \mathrm{~T}$ and $7 \mathrm{~T}$ were compared at $37^{\circ} \mathrm{C}$. However, only 4 different concentrations were used and error estimates were not provided.

The aforementioned previous studies have likewise not attempted to obtain baseline $\mathrm{T} 1$ values of whole human blood at various field strengths. T1 values of blood may be influenced by Hct, temperature, $\mathrm{pH}$, and oxygenation levels. ${ }^{9,15-19}$ In fact, accurate measurements of human blood T1 values are an important topic in itself because these values are an important MR parameter for quantitative physiological and functional MR measurements, such as arterial spin labeling with cerebral

TABLE 5. Longitudinal Relaxation Rates $R_{1}\left(\mathrm{~s}^{-1}\right)$ of Human Whole Blood at $37^{\circ} \mathrm{C}$

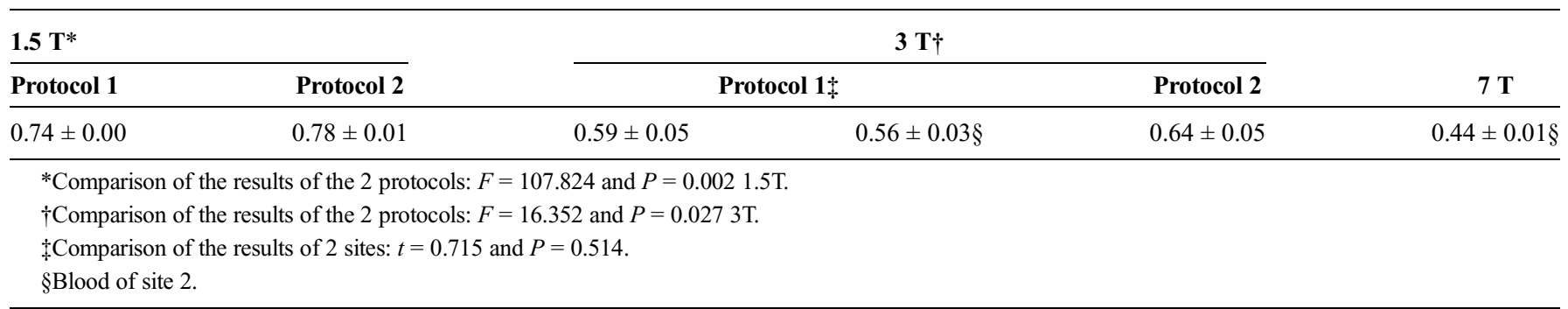



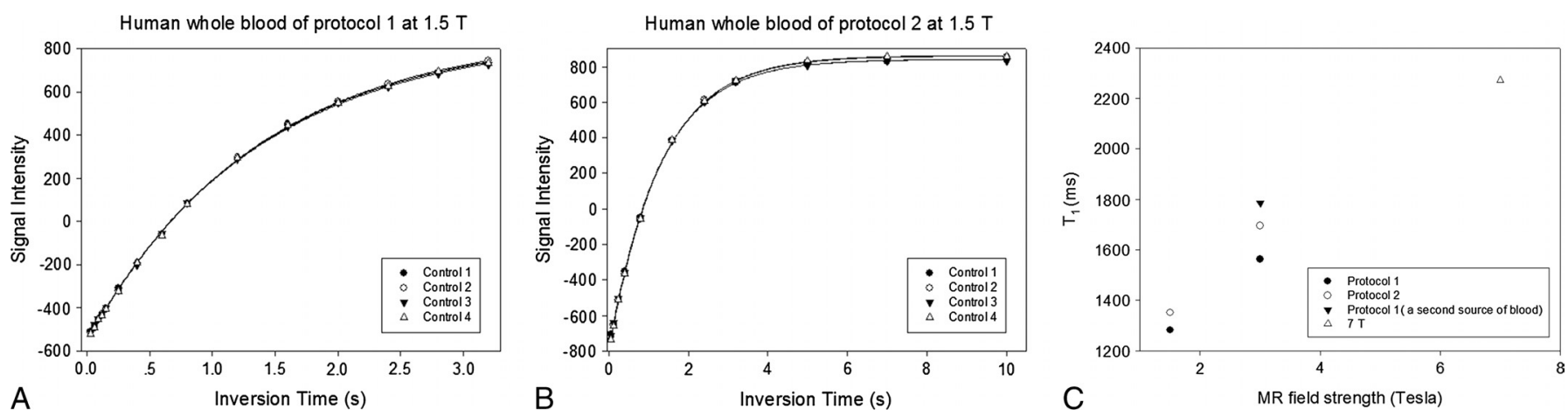

FIGURE 4. Calculation of human whole blood $R_{1}$ relaxation rate constant as performed using protocol 1 (A) and 2 (B) at $1.5 \mathrm{~T}$ as well as protocol 1 and 2 at $3 \mathrm{~T}$. Four control samples were included in the calculation. The calculated values were linearly dependent on $B_{0}(C)$.

blood volume calculations. Previously, T1 values of bovine blood had been measured at $1.5 \mathrm{~T}, 3 \mathrm{~T}^{18} 4.7 \mathrm{~T}, 7 \mathrm{~T}$, and $9.4 \mathrm{~T} .{ }^{17} \mathrm{~T} 1$ values of rat blood have also been measured at $11.7 \mathrm{~T} .{ }^{16}$ Human blood $\mathrm{T} 1$ values have also recently been measured at $3 \mathrm{~T}^{19}$ and $7 \mathrm{~T}^{15}$

Inversion recovery turbo spin-echo pulses have traditionally been the standard pulse sequences by which to measure longitudinal relaxation rate constants $\left(R_{1}\right)$. In the present study, 2 protocols were used at $1.5 \mathrm{~T}$ and $3 \mathrm{~T}$. These protocols incorporated different $\mathrm{T} 1$ times on the basis of principles outlined by Ogg and Kingsley, ${ }^{11}$ namely, that TR should be at least 3 times longer than the T1 time. Data points generated by plotting SI against TI with protocol 2 yielded data points evenly distributed along the exponential curve, whereas few data points measured with protocol 1 were distributed on the flat part of the curve (Fig. 3). Theoretically, the data from protocol 2 should give a more accurate depiction of relaxation curves for the lower concentrations, thus leading to more accurate results. Although the results from 2 protocols were slightly different from each other, no statistically significant differences were found between them, except for borderline statistically significant results with Gd-DTPA at $1.5 \mathrm{~T}$ (Table 2). The measurements of the longitudinal relaxation rate constants of human whole blood from the 2 protocols were statistically significantly different at both $1.5 \mathrm{~T}$ and $3 \mathrm{~T}$ (Table 4). Theoretically, the results from protocol 2 should be more accurate than those from protocol 1 because the former gave a relaxation curve fitting the expected exponential distribution more closely (Fig. 4). The statistically significant differences in the $R_{1}$ measurements for whole blood are likely caused by the long T1 relaxation time of whole blood and the fact that one of the protocols used a TR of 1500 milliseconds. In the end, the difference was not large enough to impact our results. We found no statistical difference when using results from protocol 1 or 2 when calculating relaxivity.

Only 1 protocol was used to measure whole blood longitudinal relaxation at $7 \mathrm{~T}$. The calculated $\mathrm{T} 1$ values of human whole blood in the present study was in accordance with the study done by Rane et $\mathrm{al}^{15}$ $(2.29 \pm 0.1$ seconds for human arterial blood, $2.09 \pm 0.12$ seconds for human venous blood, $2.16 \pm 0.1$ seconds for clinic patient's venous blood). Also, T1 values of human blood in the present study were linearly dependent on $\mathrm{B}_{0}$, which is consistent with previous studies ${ }^{15-18}$ (Fig. 4).

According to the literature, $r_{1}$ is characterized by the change in relaxation rate constant per unit concentration of $\mathrm{Gd}$ chelate and is independent of concentration. The number of relaxation rate constant data points collected is critical to derive accurate statistical predictions from the regression analysis. Only 2 concentrations $(0.25$ and $0.50 \mathrm{mM}$ ) were used for curve computation by Rohrer et $\mathrm{al}^{8}$; and 4 concentrations $(0.25,0.5,1$, and $2 \mathrm{mM})$, by Noebauer-Huhmann et al. ${ }^{14} \mathrm{In}$ the present study, 7 different concentrations were used. Slight differences are also found when obtaining relaxation rate constant measurements with the 2 used protocols. Theoretically, $R_{1}$ measured with protocol 2 should be more accurate for lower GBCA concentrations and protocol 1 for higher GBCA concentrations. Thus, it is reasonable to assume that calculations that combine data obtained using the 2 different protocols are best used to include the wide range of concentrations

TABLE 6. Comparison of $r_{1}\left(\mathrm{~s}^{-1} \cdot \mathrm{mM}^{-1}\right)$ of GBCAs With the 2 Protocols at $1.5 \mathrm{~T}$ and $3 \mathrm{~T}$

\begin{tabular}{|c|c|c|c|c|c|c|}
\hline & \multicolumn{3}{|c|}{$1.5 \mathrm{~T}$} & \multicolumn{3}{|c|}{$3 \mathbf{T}$} \\
\hline & 1 & 2 & 3 & 1 & 2 & 3 \\
\hline Gd-BOPTA & $6.15 \pm 0.38$ & $6.20 \pm 0.36$ & $6.16 \pm 0.39$ & $5.36 \pm 0.33$ & $5.37 \pm 0.33$ & $5.33 \pm 0.37$ \\
\hline Gd-DO3A-butrol & $4.58 \pm 0.19$ & $4.61 \pm 0.18$ & $4.58 \pm 0.18$ & $4.42 \pm 0.26$ & $4.46 \pm 0.24$ & $4.44 \pm 0.27$ \\
\hline Gd-DOTA & $3.89 \pm 0.14$ & $3.91 \pm 0.13$ & $3.89 \pm 0.14$ & $3.40 \pm 0.31$ & $3.43 \pm 0.29$ & $3.41 \pm 0.32$ \\
\hline Gd-DTPA & $4.22 \pm 0.34$ & $4.25 \pm 0.32$ & $4.21 \pm 0.34$ & $3.75 \pm 0.18$ & $3.76 \pm 0.17$ & $3.75 \pm 0.19$ \\
\hline Gd-DTPA-BMA & $4.46 \pm 0.09$ & $4.47 \pm 0.08$ & $4.46 \pm 0.08$ & $3.87 \pm 0.16$ & $3.89 \pm 0.15$ & $3.88 \pm 0.17$ \\
\hline Gd-DTPA-BMEA & $4.41 \pm 0.19$ & $4.43 \pm 0.18$ & $4.41 \pm 0.19$ & $4.23 \pm 0.12$ & $4.24 \pm 0.12$ & $4.23 \pm 0.12$ \\
\hline Gd-EOB-DTPA & $7.24 \pm 0.17$ & $7.24 \pm 0.15$ & $7.25 \pm 0.17$ & $5.42 \pm 0.27$ & $5.45 \pm 0.26$ & $5.42 \pm 0.27$ \\
\hline Gd-HP-DO3A & $4.32 \pm 0.49$ & $4.39 \pm 0.47$ & $4.32 \pm 0.50$ & $3.41 \pm 0.49$ & $3.46 \pm 0.46$ & $3.41 \pm 0.51$ \\
\hline
\end{tabular}

Method 1: $r_{1}$ was calculated by data gained from protocol 1 only.

Method 2: $r_{1}$ was calculated by data gained from protocol 1 and protocol 2 (concentration range, $0.0625-0.25 \mathrm{mM}$ ).

Method 3: $r_{1}$ was calculated by data gained from protocol 1 (concentration range, $0.5-4 \mathrm{mM}$ ) and protocol 2 (concentration range, $0.0625-0.25 \mathrm{mM}$ ).

GBCA indicates gadolinium-based magnetic resonance contrast agents. 

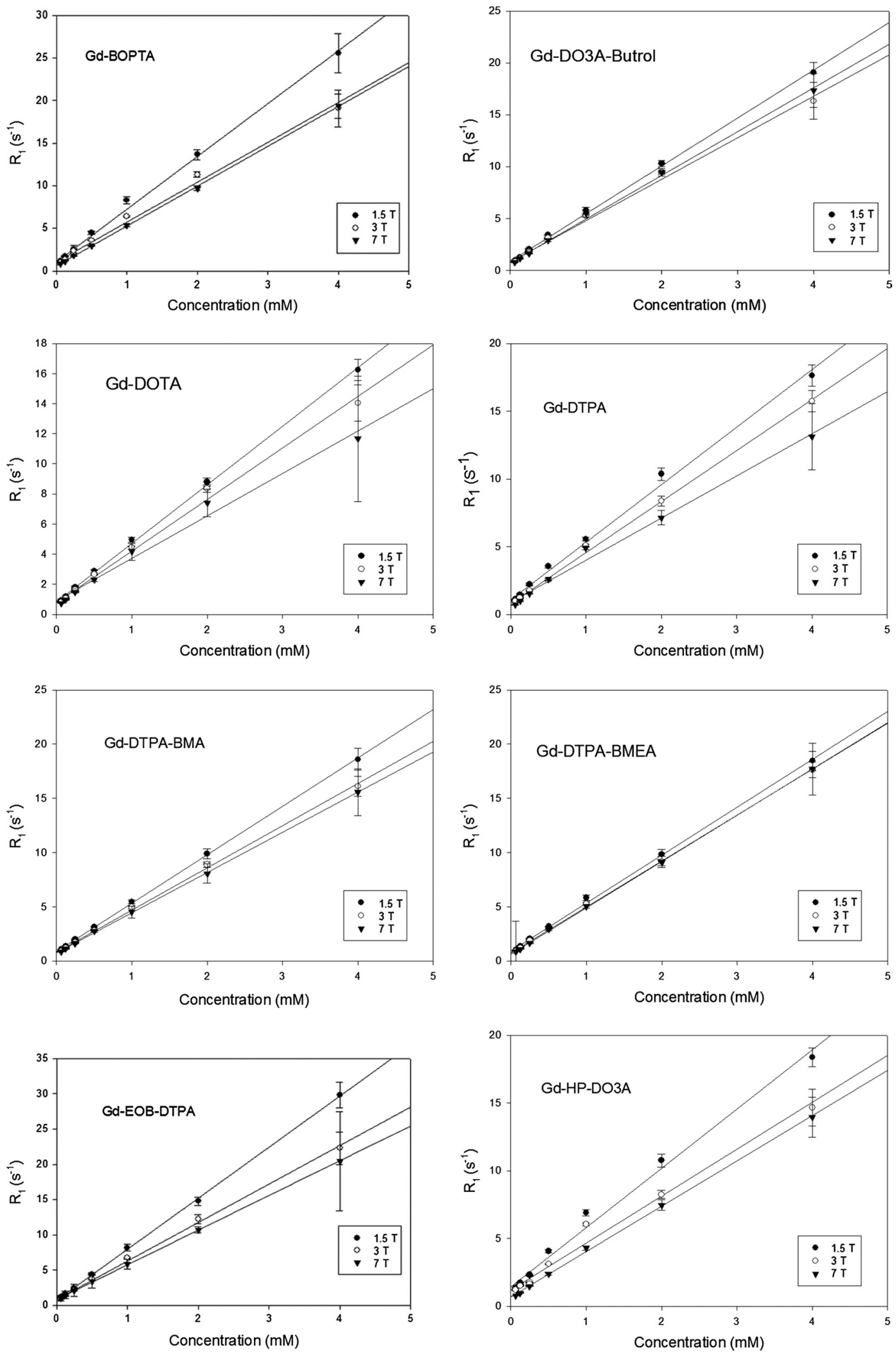

FIGURE 5. Illustrated is $R_{1}$ plotted against concentration for the $8 \mathrm{GBCAs}$ at $1.5 \mathrm{~T}, 3 \mathrm{~T}$, and $7 \mathrm{~T}$. 


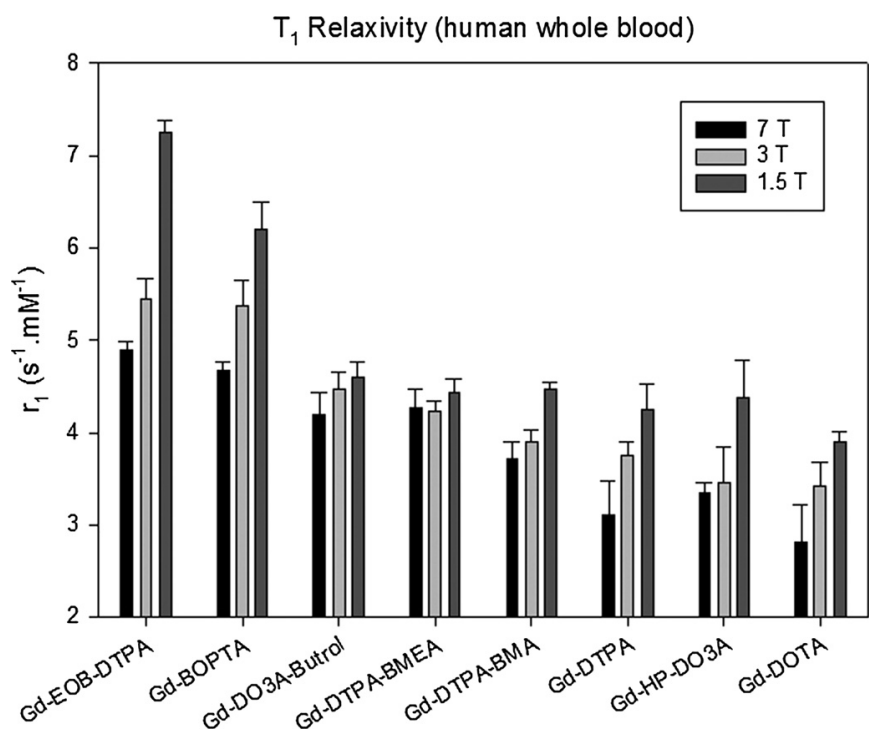

FIGURE 6. An overview of the 8 agents and their respective $r_{1}$ relaxivities at $1.5,3$, and $7 \mathrm{~T}$ is presented in a graphical format.

present. Considering this issue, 3 different methods were used to calculate $r_{1}$. The results were similar for all 3 approaches (Table 6). The calculated error (standard deviation) found from method 2 (which incorporated combined results from protocol 1 and protocol 2) was less than or equal to those calculated with the other methods; thus, we present this as our most accurate results and they are shown in Table 7. In the study of Noebauer-Huhmann et $a{ }^{14},{ }^{14}$ the small number of concentrations used for the $r_{1}$ calculation adds to the uncertainty of the results. Pintaske et $\mathrm{al}^{3}$ used a wider range of concentrations $(0.01,0.02$, plasma, however, with only 3 GBCAs. They stated that the relationship between $R_{1}$ and concentration was nonlinear with Gd-BOPTA. Noebauer-Huhmann et $\mathrm{al}^{14}$ determined that all 8 GBCAs had a nonlinear relationship between $R_{1}$ and concentration at both $3 \mathrm{~T}$ and $7 \mathrm{~T}$. In the present study, plots of $R_{1}$ against concentration of 8 GBCAs showed no consistent pattern and were not definitely linear or nonlinear (Fig. 5). Different serum total protein and albumin in each of experimental material setups could be the reason. ${ }^{20,21}$ Another reason for discrepancies can be attributed to inaccuracies of concentration determination and /or species-dependent influences of the blood plasma samples. One potential limitation of the present study is that blood oxygenation may influence blood T1 values. However, conflicting conclusions regarding the influence of blood oxygen content have been reached in previous studies. ${ }^{15-19}$ In the study of Lin et al, ${ }^{16}$ rat blood T1 values were found to be independent of oxygenation. The study of Rane et $\mathrm{al}^{15}$ demonstrated that $\mathrm{T} 1$ values of human blood at $7 \mathrm{~T}$ differed depending on whether arterial or venous blood was examined. Other limitations include the fact that citrate was present in the blood in the present study, as discussed previously in the Materials and Methods section. However, a preliminary experiment demonstrated no differences in relaxivity values on this basis, thus justifying this approach. Also, different sources of blood were used, a fact that was accounted for by controlling for Hct and ensuring that serum total protein and albumin were similar. In addition, $3 \mathrm{~T}$ measurements were obtained on samples of both the blood sources to further ensure standardization. The relaxivity values acquired at $3 \mathrm{~T}$ from both sites/samples were similar. Lower Gd concentration than what was stated on the label may also have affected our results; however, the relaxivity calculations reflect the formulation of the agents as commercially supplied (Table 2 ). $0.03,0.06,0.13,0.25,0.5,1,2,4,8,16,32$, and $64 \mathrm{mM}$ ) in human blood

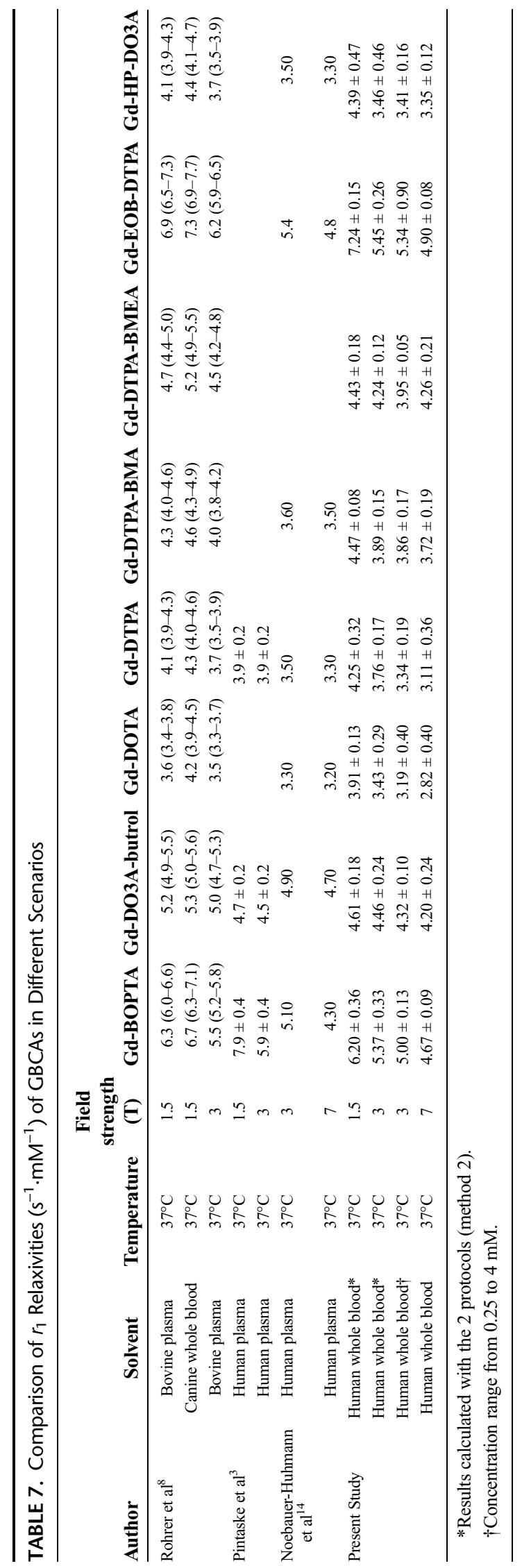

8 | www.investigativeradiology.com 
The current study provides $\mathrm{T} 1$ relaxivity measurements, obtained from human whole blood, comparing the 8 Gd chelates commonly used worldwide as intravenous contrast media for magnetic resonance, at current relevant field strengths $(1.5,3$, and $7 \mathrm{~T})$ and physiologic temperature. The results from the present study should serve as a reasonable and reliable reference for further clinical use.

\section{ACKNOWLEDGMENTS}

The authors thank Tao Ai, Regina Moritz, Xuemei Hu, John Anderson, Amir Abduljalil, PhD, Xiangyu Yang, PhD, Kellen Carrill, Jim Bankson, and Jamie Svcrek.

\section{REFERENCES}

1. Hao D, Ai T, Goerner F, et al. MRI contrast agents: basic chemistry and safety. J Magn Reson Imaging. 2012;36:1060-1071.

2. Runge VM, Ai T, Hao D, et al. The developmental history of the gadolinium chelates as intravenous contrast media for magnetic resonance. Invest Radiol. 2011; 46:807-816.

3. Pintaske J, Martirosian $\mathrm{P}$, Graf $\mathrm{H}$, et al. Relaxivity of gadopentetate dimeglumine (Magnevist), gadobutrol (Gadovist), and gadobenate dimeglumine (MultiHance) in human blood plasma at $0.2,1.5$, and 3 Tesla [published erratum appears in Invest Radiol 2006;41:859]. Invest Radiol. 2006;41:213-221.

4. Stanisz GJ, Henkelman RM. Gd-DTPA relaxivity depends on macromolecular content. Magn Reson Med. 2000;44:665-667.

5. Goetschi S, Froehlich JM, Chuck NC, et al. The protein and contrast agent-specific influence of pathological plasma-protein concentration levels on contrast-enhanced magnetic resonance imaging. Invest Radiol. 2014;49:608-619.

6. Giesel FL, von Tengg-Kobligk H, Wilkinson ID, et al. Influence of human serum albumin on longitudinal and transverse relaxation rates ( $\mathrm{r} 1$ and $\mathrm{r} 2$ ) of magnetic resonance contrast agents. Invest Radiol. 2006;41:222-228.

7. Rinck PA, Muller RN. Field strength and dose dependence of contrast enhancement by gadolinium-based MR contrast agents. Eur Radiol. 1999;9:998-1004.
8. Rohrer M, Bauer H, Mintorovitch J, et al. Comparison of magnetic properties of MRI contrast media solutions at different magnetic field strengths. Invest Radiol. 2005;40:715-724.

9. Laurent S, Elst LV, Muller RN. Comparative study of the physicochemical properties of six clinical low molecular weight gadolinium contrast agents. Contrast Media Mol Imaging. 2006;1:128-137.

10. Blockley NP, Jiang L, Gardener AG, et al. Field strength dependence of R1 and R2* relaxivities of human whole blood to ProHance, Vasovist, and deoxyhemoglobin. Magn Reson Med. 2008;60:1313-1320.

11. Ogg RJ, Kingsley PB. Optimized precision of inversion-recovery T1 measurements for constrained scan time. Magn Reson Med. 2004;51:625-630.

12. Kingsley PB, Monahan WG. Effect of increased repetition time TR on precision of inversion-recovery T(1) measurements. Magn Reson Imaging. 2001;19:279-282.

13. Lauffer RB, Parmelee DJ, Dunham SU, et al. MS-325: albumin-targeted contrast agent for MR angiography. Radiology. 1998;207:529-538.

14. Noebauer-Huhmann IM, Szomolanyi P, Juras V, et al. Gadolinium-based magnetic resonance contrast agents at 7 Tesla: in vitro $\mathrm{T} 1$ relaxivities in human blood plasma. Invest Radiol. 2010;45:554-558.

15. Rane SD, Gore JC. Measurement of T1 of human arterial and venous blood at $7 \mathrm{~T}$. Magn Reson Imaging. 2013;31:477-479.

16. Lin AL, Qin Q, Zhao X, et al. Blood longitudinal (T1) and transverse (T2) relaxation time constants at 11.7 Tesla. MAGMA. 2012;25:245-249.

17. Dobre MC, Ugurbil K, Marjanska M. Determination of blood longitudinal relaxation time (T1) at high magnetic field strengths. Magn Reson Imaging. 2007;25: 733-735.

18. Lu H, Clingman C, Golay X, et al. Determining the longitudinal relaxation time (T1) of blood at 3.0 Tesla. Magn Reson Med. 2004;52:679-682.

19. Shimada K, Nagasaka T, Shidahara M, et al. In vivo measurement of longitudinal relaxation time of human blood by inversion-recovery fast gradient-echo MR imaging at 3T. Magn Reson Med Sci. 2012;11:265-271.

20. Wang Y, Spiller M, Caravan P. Evidence for weak protein binding of commercial extracellular gadolinium contrast agents. Magn Reson Med. 2010;63:609-616.

21. Henrotte V, Vander EL, Laurent S, et al. Comprehensive investigation of the noncovalent binding of MRI contrast agents with human serum albumin. J Biol Inorg Chem. 2007;12:929-937. 